\title{
Philosophiques
}

\section{Index du volume 35 (2008)}

Volume 35, numéro 2, automne 2008

URI : https://id.erudit.org/iderudit/000538ar

DOI : https://doi.org/10.7202/000538ar

Aller au sommaire du numéro

Éditeur(s)

Société de philosophie du Québec

ISSN

0316-2923 (imprimé)

1492-1391 (numérique)

Découvrir la revue

Citer ce document

(2008). Index du volume 35 (2008). Philosophiques, 35(2), 629-630.

https://doi.org/10.7202/000538ar

Ce document est protégé par la loi sur le droit d'auteur. L'utilisation des services d'Érudit (y compris la reproduction) est assujettie à sa politique d'utilisation que vous pouvez consulter en ligne.

https://apropos.erudit.org/fr/usagers/politique-dutilisation/
Cet article est diffusé et préservé par Érudit.

Érudit est un consortium interuniversitaire sans but lucratif composé de l'Université de Montréal, l'Université Laval et l'Université du Québec à Montréal. Il a pour mission la promotion et la valorisation de la recherche. https://www.erudit.org/fr/ 


\section{Index du volume 35 (2008)}

\section{Articles}

Bilodeau, Renée, Introduction : Hommage à Joseph Nicolas Kaufmann

BrAHAMI, FrédÉRIC, Au fil conducteur du scepticisme: science et métaphysique chez Glanvill

BRANDAO, Rodrigo, Voltaire et le scepticisme

Cavaillé, Jean-Pierre, "Je doute qui je suis, je me perds, je m'ignore». Ego baroque et sujet cartésien

Charles, Sébastien, Introduction. Le scepticisme à l'âge classique: enjeux et perspectives

Charles, Sébastien, De Popkin à Rousseau : retour sur le scepticisme des Lumières

Courtois, StÉPHAne, Le cognitivisme moral de Habermas fait-il face au problème de Frege-Geach?

Couture, Jocelyne, Sélection rationnelle

Cumin, David, Freud et la guerre

Currie, Gregory, L'ironie : echo ou feintise?

Dias, MaRia C., Relier la conscience phénoménale et éliminer le fossé explicatif $5 \mathrm{I} 3$

Duval, SOPHIE, "Pouvoir réfléchissant " et "force ascensionnelle »: la dynamique régressive de l'ironie

ENGEL, PASCAL, Introduction: raillerie, satire, ironie et sens plus profond

Franceschi, Paul, Une défense logique du modèle de Maher pour les délires polythématiques

IPPERCIEL, DONALD, Communautés morales et universalisme: Quelles sont les responsabilités morales des individus des pays riches envers les pays pauvres?

Kaufmann, J. Nicolas, Critique du programme de naturalisation en philosophie de l'esprit

LaUrsen, John S., Scepticisme et cynisme dans l'œuvre de Pierre de Valence

LeClercQ, Bruno, Les données immédiates de la conscience. Neutralité métaphysique et psychologie descriptive chez James et Husserl

Lennon, Thomas N., La réponse de Régis à Huet concernant le doute cartésien

Lombardo, Patrizia, Tendresse et pudeur chez Stendhal

Maia Neto, José R., Huet sceptique cartésien

Mestiri, SOUMAYA, Identité procédurale ou substantielle?

Mulligan, Kevin, Ironie, valeurs cognitives et bêtise

Paganini, Gianni, Montaigne, Estienne et l'invention de l'apparence

Plourde, Jimmy, Du réalisme des Recherches logiques 


\section{$630 \cdot$ Philosophiques / Automne 2008}

Reboul, Anne, L'ironie auctoriale: une approche gricéenne est-elle possible? $\quad 25$

ROY, OLIVIER, Intentions rationnelles et acceptation dans les délibérations $\quad 525$

Sacrini A. Ferraz, Markus, Perception et culture chez Merleau-Ponty 297

Sousa Melo, Candida, Controverse sur la causalité mentale dans l'action $\quad 345$

\section{Disputationes}

Chauvier, StÉPhane, Volitions et auto-affection I3 I

Laurier, Daniel, Sommes-nous tous des épiphénomènes II9

Livet, PIERre, Volition, ajustements moteurs et exploration des valeurs $\quad$ I 27

Proust, Joëlle, Précis de La Nature de la Volonté IO9

Proust, Joëlle, Réponses à mes critiques $\quad$ I39

\section{Comptes rendus}

Bunge, Mario, Chasing Reality: Strife over Realism (Davy Mougenot) 609

Peckhaus, Volker (Hrsg.) Oskar Becker und die Philosophie der

$\begin{array}{ll}\text { Mathematik (Yvon Gauthier) 6I2 } & \end{array}$

Peirce, Charles S., Écrits logiques, Tiercelin Claudine et Thibaud Pierre (dir.), $\begin{array}{ll}\text { CEuvres III (Olivier Perru) } & 620\end{array}$

Ramsey, William M., Representation Reconsidered (Pierre Steiner) 6I6

Tieszen, Richard, Phenomenology, Logic and the Philosophy of Mathematics (Yvon Gauthier) 